\title{
Strategy-making for a Proactive Distribution Company in the Real-time Market with Demand Response
}

\author{
Chunyu Zhanga ${ }^{\mathrm{a}}$, Qi Wang ${ }^{\mathrm{b}}$, Jianhui Wang ${ }^{\mathrm{c}, *}$, Magnus Korpås ${ }^{\mathrm{a}}$, Mohammad E. \\ Khodayar $^{\text {d }}$ \\ ${ }^{a}$ Department of Electric Power Engineering, Norwegian University of Science and \\ Technology, Trondheim, Norway \\ ${ }^{b}$ Center for Electric Power and Energy, Technical University of Denmark, Lyngby, \\ Denmark \\ ${ }^{c}$ Energy Systems Division, Argonne National Laboratory, Argonne, IL, USA \\ ${ }^{d}$ Electrical Engineering Department, Southern Methodist University, Dallas, TX, USA
}

\begin{abstract}
This paper proposes a methodology to optimize the trading strategies of a proactive distribution company (PDISCO) in the real-time market by mobilizing the demand response. Each distribution-level demand is considered as an elastic one. To capture the interrelation between the PDISCO and the real-time market, a bi-level model is presented for the PDISCO to render continuous offers and bids strategically. The upper-level problem expresses the PDISCO's profit maximization, while the lower-level problem minimizes the operation cost of the transmission-level real-time market. To solve the proposed model, a primal-dual approach is used to translate this bi-level model into a single-level mathematical program with equilibrium constraints. Results of case studies are reported to show the effectiveness of the proposed model.
\end{abstract}

Keywords: Demand response (DR), proactive distribution company (PDISCO), multi-period AC power flow, mathematical program with equilibrium constraints (MPEC), mathematical program with primal and dual constraints (MPPDC).

\footnotetext{
* Corresponding author

Email addresses: chunyu.zhang@ntnu.no (Chunyu Zhang), qiwa@elektro.dtu.dk (Qi Wang), jianhui.wang@anl.gov (Jianhui Wang), magnus.korpas@ntnu.no (Magnus Korpås), mkhodayar@smu.edu (Mohammad E. Khodayar)
}

Preprint submitted to Applied Energy

July 26, 2016

(C) 2016. This manuscript version is made available under the Elsevier user license http://www.elsevier.com/open-access/userlicense/1.0/ 


\section{Nomenclature}

Sets and Indices

$i, j, B^{D S}$

$n, m, B^{T S}$

$i j, \Lambda^{D S}$
$n m, \Lambda^{T S}$

$l, L$

$d, D$

$g, G$

$t, T$

$\mathcal{M}_{L}, \mathcal{M}_{D}$

$\mathcal{M}_{G}$

Variables

$r_{t g}^{U P}, r_{t g}^{D N}$

$P_{t I}^{D R T}, Q_{t I}^{D R T}$

$P_{t d}^{S T}$

$P_{t l}^{S D}, Q_{t l}^{S D}$

$Q_{t i}^{C}$

$P_{t, i j}^{\text {Flow }}, Q_{t, i j}^{\text {Flow }}$

$V_{t i}$
Index and set of distribution-level (DL) and transmission-level (TL) buses, respectively.

Index and set of DL feeders and TL lines, respectively.

Index and set of DL demands and TL demands, respectively.

Index and set of TL generators.

Index and set of time periods (e.g., hours per day).

Mapping of the set of DL/TL demands onto the set of DL/TL buses, respectively.

Mapping of the set of TL generators onto the set of TL buses.

Real-time up and down regulation power of generator $g$ at time $t[\mathrm{MW}],[\mathrm{MW}]$.

Real-time offering/bidding quantities of the PDISCO at time $t$ [MW], [MVar].

TL load-shedding of demand $d$ at time $t$ [MW].

Active and reactive power of DS load-shedding for demand $l$ at time $t[\mathrm{MW}],[\mathrm{MVar}]$.

Real-time reactive power from DL shunt compensator at bus $i$ at time $t$ [MVar].

Real-time active and reactive power flows through DL feeder $i j$ at time $t[\mathrm{MW}],[\mathrm{MVar}]$.

Real-time voltage magnitude of DL bus $i$ at time $t[\mathrm{kV}]$. 


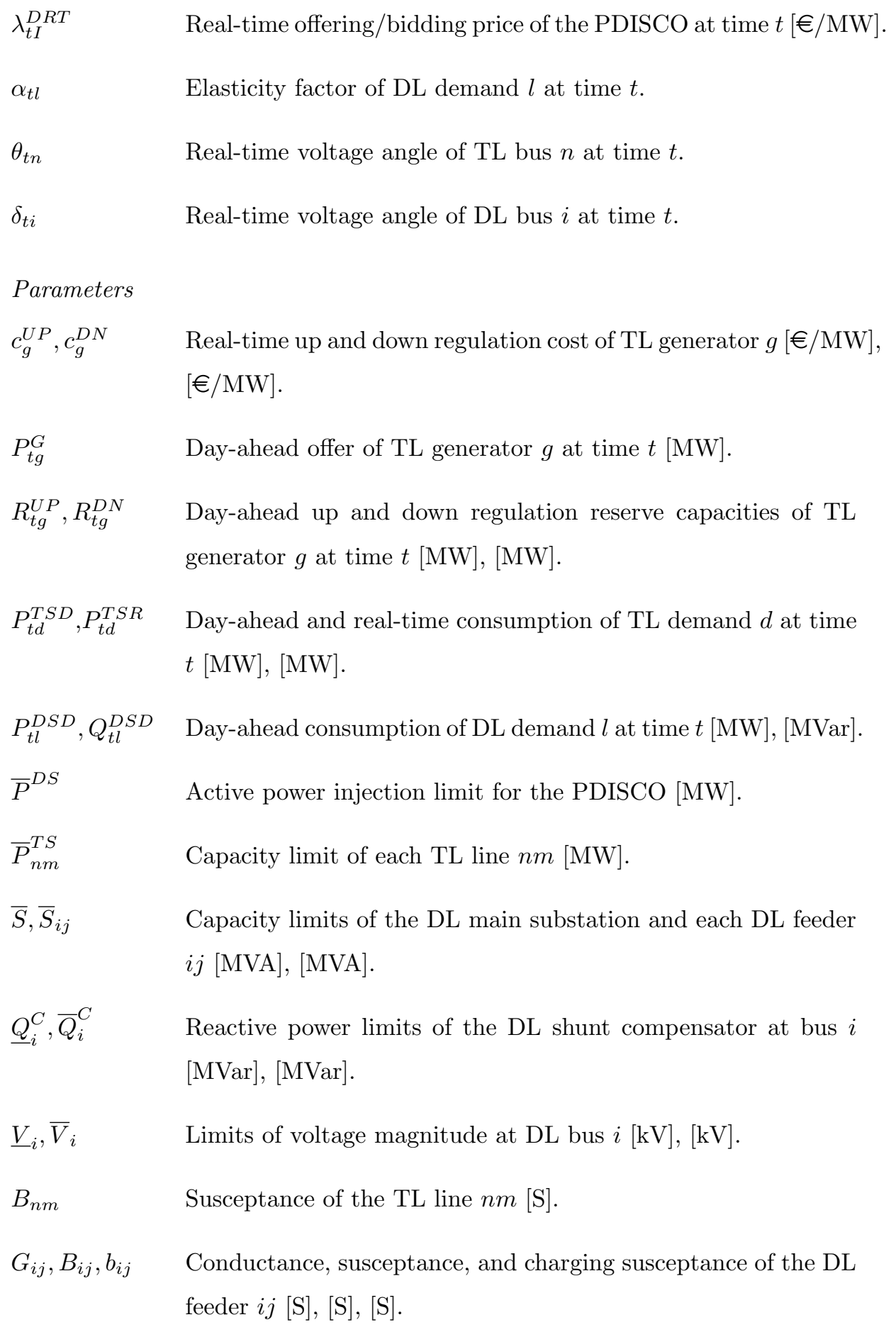




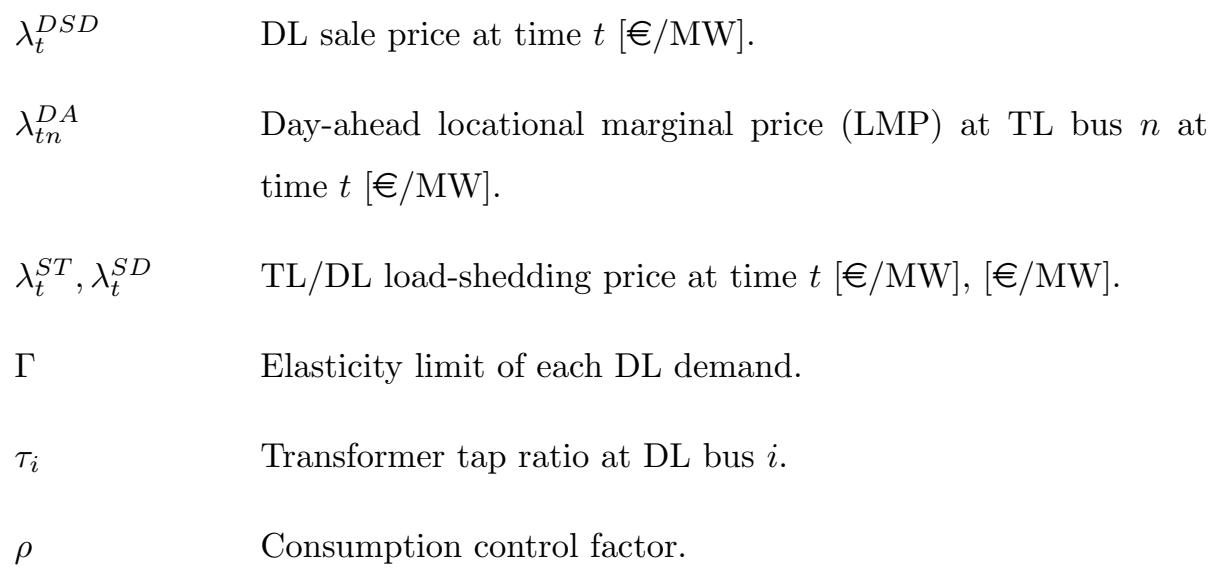

\section{Introduction}

A low-cost and high-efficiency demand response (DR) has been recognized worldwide as a valuable resource [1, 2]. Because DR is dispersed and highly sensitive to the real-time price [3, 4], an aggregator is commonly considered as a third entity to launch direct control or incentive schemes to manage individual DRs [5]. As addressed in the New York Reforming Energy Vision (NY REV) [6], the concept of Distributed System Platform Provider (DSPP) is raised to promote the utilization of distributed energy resources (DERs) deployed in the distribution-level (DL) network. To enhance the transmission and distribution trading efficiency, the distribution company (DISCO) can bee seen as a DSPP, while DR [7, 8], distributed generation (DG) [9], and microgrids (MGs) [10] can be considered as the major flexibility providers [11, 12, 13. In practice, the DISCO has full knowledge of both the transmission-level (TL) markets and the DL operations. For this reason, the DISCO is a superior representation [14, 15] of the DR resources available to trade in the TL market.

The well-developed smart grid technology [16, 17] that exists today turns bidirectional power exchanging between the distribution and transmission networks into reality. As a profit-driven company, to participate in the real-time market, at each time $t$, the DISCO has to downwardly optimize the DR portfolio and upwardly hand over a trading strategy (offer/bid) to the wholesale market 
for profit maximization. The continuous strategic behaviors of the DISCO result in a dual role in the market, i.e., as an active producer when providing offers, but as an active consumer when submitting bids. Accordingly, the trading between the DISCO and the TL market features in a bidirectional transaction. To characterize this type of DISCO, we define it as a proactive DISCO (PDISCO) in this paper.

Note that the PDISCO's trading strategies are correlated with the market's outcomes. Thus, the PDISCO trading within the market can be formulated as a bi-level game-theoretic model. The lower-level problem is about the realtime market clearing based on DC power flow, aiming to minimize the TL operation cost. The upper-level problem is to maximize the PDISCO's profit by $\mathrm{AC}$ power flow constraints, with the PDISCO seeking to render strategic offers/bids. It is also worth noting that the lower-level problem is linear and thus convex, while the upper-level problem is non-linear and non-convex. A primaldual approach [18] can be used to reformulate the proposed bi-level model as a solvable mathematical program with equilibrium constraints (MPEC).

Some papers pertaining to DISCO trading in the TL markets are available in the literature. In the day-ahead market, a bi-level model is proposed in [19] to address a DISCO's operation decisions with DGs and interruptible loads (ILs). However, the DISCO's offers or bids are not included in the market's objective. A hierarchical market structure is provided in [20] to depict the interaction between the distribution and transmission networks. On the basis of this structure, a bi-level model is presented, while the locational marginal prices (LMPs) are not endogenously obtained by the power flow constraints. To address a DL market framework by incorporation into the TL market, a bilevel model is proposed for a load serving entity (LSE) to manage aggregatorbased DR through a dynamic pricing mechanism [21]. In particular, DC power flow is used to model the distribution network. For simplicity, the TL market performance and physical constraints are not fully considered. At the dayahead stage, a static bi-level model [22] is presented for the DISCO's make energy acquisitions from the TL wholesale market, ILs and dispatchable DGs. 
A multi-period bi-level model is proposed and further developed in [23, to indicate the market impacts of the ILs and the DISCO-owned DGs. However, the network constraints are ignored.

From differing perspectives, bi-level modeling is increasingly used to elaborate the trading strategies in markets [24]. In a pool market, a bi-level model is proposed in 25] for a strategic producer to use against its rivals. A stage-based stochastic bi-level model is presented in [26] to derive the offering strategy for a wind-power producer that is involved in both the day-ahead market and the balancing market. From the consumer side, a multi-period bilevel model [27] is proposed to minimize the payment in Pool markets according to LMPs. To make up strategic bidding curves for a large consumer, a bi-level model is further reported in [28] to minimize its day-ahead payment. The strategic operation between the DL operator and MGs is implemented by a bi-level model in [29]. In addition, the authors in [30] propose a bi-level model to handle multiple MGs in a competitive environment.

On the other hand, to enable flexible demand trading in existing electricity markets, load shifting is achieved by a Lagrangian relaxation-based heuristic approach [31. With the presented market mechanism, the DR performance is further validated and discussed by 32 . The network constraints are excluded by the model. From the management perspective, a contract-based cluster 33 . is proposed to facilitate elastic demands to purchase or sell energy according to the interactions between DG and the main grid. The authors also ignore the network influence. To incorporate the price-responsive demand in multi-period energy scheduling, as indicated in 34, the consumer payment minimization is ensured in a pool market trading. Although the TL network is modeled as constraints, the consumer deployment is not exhibited by the DL network. In [35], a hierarchical DR bidding framework is proposed to engage in the day-ahead energy markets. In order to minimize the disutility of aggregated consumers, a simple uniform-price market mechanism is featured in [36] to allocate a certain amount of load adjustments to individual demands. For load reduction, the hourly strategies of the independent system operator (ISO) include load shifting 
and curtailment, the use of onsite generation, and energy storage systems. A demand-side virtual power plant (VPP) is presented to be a source of spinning reserves in [37]. Spatially-distributed load groups are optimized by a regional load aggregation scheme. At day-ahead stage, in regard to DR resources participating in both energy and reserve scheduling, a robust model is given in [38] to schedule smart distribution network to immunize uncertainties. Aiming to mitigate the impact of wind power variability, an agent-based modeling framework is used to represent hierarchical power systems [39], and a DR aggregator is employed by a wind power producer to make offering strategies [40]. In view of control schemes, thermostatic loads are aggregated to manage frequency and energy imbalances in power systems [4]. Based on a dynamic control model, the valuation of a thermal storage-enabled DR is obtained in [42]. In [43], a

direct-load control algorithm is presented to aggregate the water heaters for providing regulation service.

Considering the context above, the contributions of this paper are threefold:

1) Present a framework for the PDISCO to trade in the real-time market.

2) Propose a bi-level model for the PDISCO to continuously derive strategic offers and bids by optimizing DR portfolios.

3) Define an elasticity factor to represent the DR resource.

The rest of this paper is organized as follows: A trading framework is presented in Section 2 for the PDISCO participating in the TL real-time market with DL DRs. A bi-level model and solving approach are proposed in Section 3 to embody the interrelations between the PDISCO and the TL market. In Section 4, case studies are performed and sensitive parameters are analyzed. Finally, conclusions are presented in Section 5.

\section{PDISCO Trading Framework}

As addressed above, the PDISCO gets an opportunity to strategically participate in the TL real-time market by dynamically managing the DL dispersed DRs. 


\section{1. $P D I S C O$}

At the transmission level, the day-ahead market is cleared prior to the realtime market. Assuming a PDISCO is located at transmission bus $I$, its dayahead purchases $\left(\lambda_{t I}^{D A}, P_{t I}^{T S D}\right)$ and demands $\left(P_{t l}^{D S D}\right)$ are fixed at the very beginning of the next day. Throughout the day, as a competitive market player, at each time $t$, the PDSICO upwardly sells/purchases power via a strategic offer/bid $\left(\lambda_{t I}^{D R T}, P_{t I}^{D R T}\right)$ in the real-time market, and downwardly schedules the elasticity (DR) of each DL demand $l$ to meet its requirements.

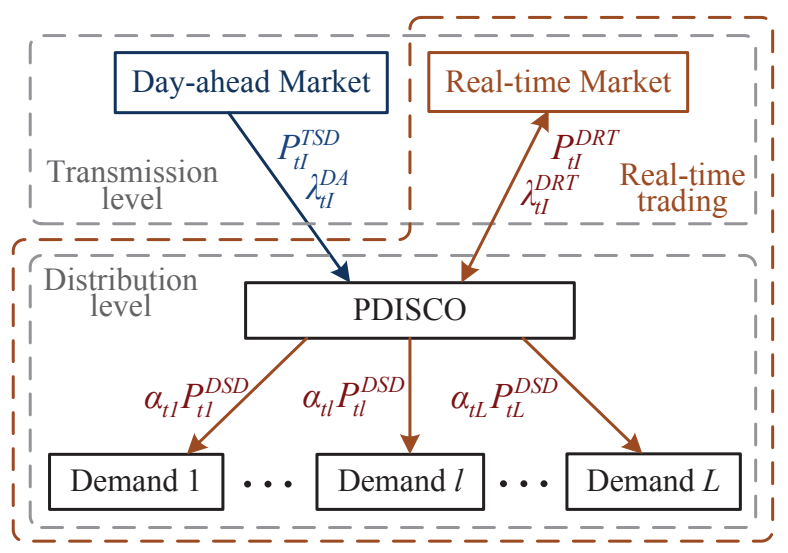

Figure 1: Real-time trading framework of the PDISCO.

As shown in Fig. 1, to maximize profit, the PDISCO's offering/bidding quantity $P_{t I}^{D R T}$ is determined by optimizing the real-time DR portfolio against the physical constraints of the distribution network. To identify the continuous offers and bids, at each time $t$ : If $P_{t I}^{D R T} \geq 0$, the PDISCO acts as an active producer with a lower offering price $\lambda_{t I}^{D R T}$; If $P_{t I}^{D R T}<0$, the PDISCO behaves as an active consumer with a higher bidding price $\lambda_{t I}^{D R T}$.

\subsection{Demand response}

In this paper, in order to specify the dispersed DR resources, $\alpha_{t l}$ is used to represent the elasticity with the limit $\Gamma(\Gamma \geq 1)$, which characterizes the load-shifting capability of real-time demand at time $t$. Therefore, the real-time 


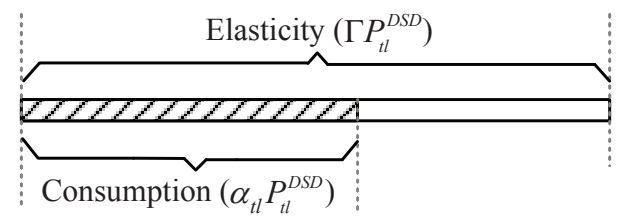

Figure 2: Definition of demand response.

consumption at DL bus $l$ can be seen as $\alpha_{t l} P_{t l}^{D S D}$, as shown in Fig. 2. In realtime operation, the PDISCO passes the $\alpha_{t l}$ schedules to individual demands on the basis of system requirements, and each demand takes responsibility for executing the related schedule. To indicate the behaviors of DR $l$, at each time $t$ : If $0 \leq \alpha_{t l}<1$, demand $l$ reduces the consumption as required at the day-ahead stage. This implies that the declined portion $\left(1-\alpha_{t l}\right) P_{t l}^{D S D}$ can be reallocated to the other time periods; If $1 \leq \alpha_{t l} \leq \Gamma$, demand $l$ consumes more than its dayahead prediction. This means the increasing portion $\left(\alpha_{t l}-1\right) P_{t l}^{D S D}$ is committed to accommodate the shifted loads from the other time slots.

\section{Problem Formulation}

\subsection{Assumptions}

The formulation of the proposed bi-level model is based on the following assumptions:

1) Only a single PDISCO is considered to participate in the real-time market.

2) The PDISCO operates the distribution network, manages individual DRs, and interconnects with the TL network through one main substation.

3) The active power is eligible to be traded in the real-time market, since no uniform reactive power market is acknowledged. The reactive power exchanging is assumed to be balanced at the DL main substation.

4) We assume the PDISCO can explicitly anticipate the impacts of its strategic offers/bids, versus the market's outcomes. One offer/bid is allowed per time $t$. 


\subsection{Bi-level model}

As indicated in Section 2, the transactions between the PDISCO and the real-time market are characterized in a bidirectional fashion. The PDISCO's trading strategies (offering/bidding prices and power quantities) are endogenously interrelated with the market's outcomes (LMPs and production/consumption quantities). To highlight these features, the PDISCO's strategy-making problem can be formulated as a bi-level game-theoretic model [18]. The lower-level problem (1) seeks to achieve cost minimization during real-time TL balancing. The upper-level problem (2) obtains the offering/bidding strategies of the PDISCO by minimizing its minus-profit.

1) Lower-level real-time market problem.

$$
\begin{gathered}
\underset{\Xi R T T}{\operatorname{Minimize}} \sum_{t, g}\left(c_{g}^{U P} r_{t g}^{U P}-c_{g}^{D N} r_{t g}^{D N}\right) \\
+\sum_{t} \lambda_{t I}^{D R T} P_{t I}^{D R T}+\sum_{t, d} \lambda_{t}^{S T} P_{t d}^{S T}
\end{gathered}
$$

s.t.

For the PDISCO's location bus $I$ :

$$
\begin{aligned}
& \sum_{(g: I) \in \mathcal{M}_{G}}\left(P_{t g}^{G}+r_{t g}^{U P}-r_{t g}^{D N}\right)+P_{t I}^{D R T} \\
= & \sum_{I m \in \Lambda^{T S}} B_{I m}\left(\theta_{t I}-\theta_{t m}\right): \lambda_{t I}^{R T}, \forall t \\
- & \bar{P}^{D S} \leq P_{t I}^{D R T} \leq \bar{P}^{D S}: \beta_{t}^{-}, \beta_{t}^{+}, \forall t
\end{aligned}
$$

For the other TL buses:

$$
\begin{aligned}
& \sum_{(g: n) \in \mathcal{M}_{G}}\left(P_{t g}^{G}+r_{t g}^{U P}-r_{t g}^{D N}\right)-P_{t d}^{T S R}+P_{t d}^{S T} \\
= & \sum_{n m \in \Lambda^{T S}} B_{n m}\left(\theta_{t n}-\theta_{t m}\right): \lambda_{t n}^{R T}, \forall t, n_{(d: n) \in \mathcal{M}_{D}} \\
0 \leq & r_{t g}^{U P} \leq R_{t g}^{U P}: \phi_{t g}^{-}, \phi_{t g}^{+}, \forall t, g \\
0 \leq & r_{t g}^{D N} \leq R_{t g}^{D N}: \psi_{t g}^{-}, \psi_{t g}^{+}, \forall t, g \\
0 \leq & P_{t d}^{S T} \leq P_{t d}^{T S R}: \nu_{t d}^{-}, \nu_{t d}^{+}, \forall t, d \\
- & \bar{P}_{n m}^{T S} \leq B_{n m}\left(\theta_{t n}-\theta_{t m}\right) \leq \bar{P}_{n m}^{T S}: \mu_{t, n m}^{-}, \mu_{t, n m}^{+},
\end{aligned}
$$




$$
\begin{aligned}
& \forall t, n m \in \Lambda^{T S} \\
& -\pi \leq \theta_{t n} \leq \pi: \sigma_{t n}^{-}, \sigma_{t n}^{+}, \forall t, n \\
& \theta_{t 1}=0: \zeta_{t}, \forall t
\end{aligned}
$$

where $\Xi^{R T T}=\left\{r_{t g}^{U P}, r_{t g}^{D N}, P_{t I}^{D R T}, P_{t d}^{S T}, \theta_{t n}\right\}$ is the variable set of the lower-level problem.

The real-time market is cleared by (1). The objective function (1a) is to minimize the TL operation cost, which covers the regulation cost of each generator $g$, the strategic offers/bids of the PDISCO, and the cost of load-shedding invocations. DC power flow is used to embody the TL operation conditions. At each time $t$ : Constraints $(1 \mathrm{~b})$ and $(1 \mathrm{~d})$ guarantee the power balance at the PDISCO's location bus $I$ and the other TL buses, respectively. Constraints (1c) identify the bounds of the PDISCO's offering/bidding quantity $P_{t I}^{D R T}$. Constraints (1e) and (1f) express the up and down regulation $\left(r_{t g}^{U P}, r_{t g}^{D N}\right)$ of each generator, limited by its up and down reserve capacity $\left(R_{t g}^{U P}, R_{t g}^{D N}\right)$, respectively. Constraints (1g) impose the TL load-shedding limits. Constraints (1h) specify the capacity limits of the TL line $n-m$. Constraints (1i) limit the real-time TL voltage angle at bus $n$. Constraints $(1 \mathrm{j})$ set the TL bus 1 as the reference bus. In addition, the dual variables for each group of constraints are indicated at the right side of a colon.

2) Upper-level PDISCO problem.

$$
\begin{gathered}
\underset{\substack{\Xi R T T \\
\cup \Xi P D I S C O \cup \Xi D \text { ual } \\
\operatorname{Minimize}}}{ } \sum_{t} \lambda_{t I}^{D A} P_{t I}^{T S D}-\sum_{t} \lambda_{t I}^{R T} P_{t I}^{D R T} \\
+\sum_{t, l} \lambda_{t}^{S D} P_{t l}^{S D}-\sum_{t, l} \lambda_{t}^{D S D} \alpha_{t l} P_{t l}^{D S D}
\end{gathered}
$$

s.t.

Elasticity rate of each demand:

$0 \leq \alpha_{t l} \leq \Gamma, \forall t, l$

For the PDISCO's reference bus 1 (i.e., TL bus $I$ ):

$$
P_{t I}^{T S D}-P_{t I}^{D R T}-\alpha_{t 1} P_{t 1}^{D S D}+P_{t 1}^{S D}
$$




$$
\begin{aligned}
& =\sum_{1 j \in \Lambda^{D S}} P_{t, 1 j}^{F l o w}, \forall t \\
& Q_{t I}^{T S D}-Q_{t I}^{D R T}+Q_{t 1}^{C}-\alpha_{t 1} Q_{t 1}^{D S D}+Q_{t 1}^{S D} \\
& =\sum_{1 j \in \Lambda^{D S}} Q_{t, 1 j}^{F l o w}, \forall t \\
& V_{t 1}=1, \forall t \\
& \delta_{t 1}=0, \forall t \\
& \left(P_{t I}^{T S D}-P_{t I}^{D R T}\right)^{2}+\left(Q_{t I}^{T S D}-Q_{t I}^{D R T}\right)^{2} \leq \bar{S}^{2}, \forall t
\end{aligned}
$$

For the other DL buses:

$$
\begin{aligned}
& -\alpha_{t l} P_{t l}^{D S D}+P_{t l}^{S D}=\sum_{i j \in \Lambda^{D S}} P_{t, i j}^{F l o w}, \forall t, l_{(l: i) \in \mathcal{M}_{l}} \\
& -\alpha_{t l} Q_{t l}^{D S D}+Q_{t l}^{C}+Q_{t l}^{S D}=\sum_{i j \in \Lambda^{D S}} Q_{t, i j}^{F l o w}, \forall t, l_{(l: i) \in \mathcal{M}_{l}} \\
& P_{t, i j}^{\text {Flow }}=-\tau_{i} V_{t i}^{2} G_{i j}+V_{t i} V_{t j}\left[G_{i j} \cos \left(\delta_{t i}-\delta_{t j}\right)\right. \\
& \left.+B_{i j} \sin \left(\delta_{t i}-\delta_{t j}\right)\right], \forall t, i j \in \Lambda^{D S} \\
& Q_{t, i j}^{\text {Flow }}=\tau_{i} V_{t i}^{2} B_{i j}-0.5 b_{i j}+ \\
& V_{t i} V_{t j}\left[G_{i j} \sin \left(\delta_{t i}-\delta_{t j}\right)-B_{i j} \cos \left(\delta_{t i}-\delta_{t j}\right)\right], \\
& \forall t, i j \in \Lambda^{D S} \\
& \left(P_{t, i j}^{\text {Flow }}\right)^{2}+\left(Q_{t, i j}^{\text {Flow }}\right)^{2} \leq\left(\bar{S}_{i j}\right)^{2}, \forall t, i j \in \Lambda^{D S} \\
& 0 \leq P_{t l}^{S D} \leq P_{t l}^{D S D}, \forall t, l \\
& 0 \leq Q_{t l}^{S D} \leq Q_{t l}^{D S D}, \forall t, l \\
& P_{t l}^{D S D} Q_{t l}^{S D}-P_{t l}^{S D} Q_{t l}^{D S D}=0, \forall t, l \\
& \underline{Q}_{i}^{C} \leq Q_{t i}^{C} \leq \bar{Q}_{i}^{C}, \forall t, i \\
& \underline{V_{i}} \leq V_{t i} \leq \overline{V_{i}}, \forall t, i \\
& -\pi \leq \delta_{t i} \leq \pi, \forall t, i \\
& \sum_{t, l} \alpha_{t l} P_{t l}^{D S D}-\sum_{t, l} P_{t l}^{S D}=\rho \sum_{t} P_{t I}^{T S D}, \\
& \lambda_{t I}^{R T}, P_{t I}^{D R T} \in \arg (R T T)
\end{aligned}
$$

where $\Xi^{P D I S C O}=\left\{\lambda_{t I}^{D R T}, Q_{t I}^{D R T}, \alpha_{l t}, P_{t l}^{S D}, Q_{t l}^{S D}, P_{t, i j}^{F l o w}, Q_{t, i j}^{F l o w}, Q_{t i}^{C}, \delta_{t i}, V_{t i}\right\}$ is 
the variable set of the upper-level PDISCO problem. $\Xi^{D U A L}$ is the set of dual variables including the LMPs $\lambda_{t n}^{R T}$ of the real-time market.

The objective (2a) of the upper-level problem is to minimize the PDISCO's minus-profit (maximize profit), which comprises the purchases from the dayahead market, the revenue/cost from the PDISCO's strategic offers/bids in the real-time market, the penalty of possible DL load-shedding, and the revenue from selling electricity to the DL demands. The PDISCO's main substation, located at the TL bus $I$, is seen as the DL reference bus 1 . AC power flow is used to formulate the PDISCO's operation model. At each time $t$ : Constraints (2b) limit the elasticity rate of each DL demand. Constraints (2c) and (2d) realize the AC power balance at the DL reference bus. Constraints (2e), (2f), (2q) and (2r) enforce the value of voltage magnitude and angle at each bus $i$. Constraints (2g), (2l) and (2p) impose the capacity limits of the main substation, each feeder, and each compensator, respectively. Constraints (2h) and (2i) ensure the AC power balance at the other DL buses. Constraints $(2 \mathrm{j})$ and $(2 \mathrm{k})$ represent the AC power flow through feeder $i-j$. Constraints (2m)-(2o) keep the demand power factor constant if DL load-shedding occurs. Constraints (2s) state consumption control over the whole time span (e.g., 24 hours per day) with the factor $\rho$. For instance, when $\rho=1$, these constraints guarantee that the consumption of each demand across the whole time span should be equal to the same amount purchased by the PDISCO from the day-ahead market. In other words, constraints (2s) ensure load shifting between the hours while maintaining the total consumption at a certain level. Note that the PDISCO's offering/bidding price $\lambda_{t I}^{D R T}$ is the upper-level decision variable treated as a parameter in the lower-level problem. Constraints (2t) represent the interactive impacts between the PDSICO's trading strategies and the market's outcomes.

\section{3. $M P E C$}

The linearity of the lower-level problem allows the proposed bi-level problem to be transformed into a single-level MPEC by replacing the lower-level problem with Karush-Kuhn-Tucker (KKT) conditions or a primal-dual approach. 
As addressed in [18, 44, the primal-dual approach is more efficient than KKT conditions for off-the-shelf branch-and-cut software. Thus, the primal-dual approach is adopted as the alternative method, which renders a mathematical program with primal and dual constraints (MPPDC).

The MPPDC for the lower-level real-time market problem is indicated in (3). Constraints (3a)-(3k) are the dual constraints of the primal constraints (1b)-(1j). Constraint (3l) is the associated strong duality equality constraint, which ensures the equality of the primal and dual objective values.

$$
\begin{aligned}
& c_{g}^{U P}+\lambda_{t n}^{R T}+\phi_{t g}^{+}-\phi_{t g}^{-}=0, \forall t, g_{(g: n) \in \mathcal{M}_{G}} \\
& -c_{g}^{D N}-\lambda_{t n}^{R T}+\psi_{t g}^{+}-\psi_{t g}^{-}=0, \forall t, g_{(g: n) \in \mathcal{M}_{G}} \\
& \lambda_{t I}^{D R T}+\left(\lambda_{t I}^{R T}\right)_{n=I}+\beta_{t}^{+}-\beta_{t}^{-}=0, \forall t \\
& \lambda_{t}^{S T}+\lambda_{t n}^{R T}+\nu_{t d}^{+}-\nu_{t d}^{-}=0, \forall t, d_{(d: n) \in \mathcal{M}_{D}} \\
& \sum_{n m \in \Lambda^{T S}} B_{n m}\left(\lambda_{t n}^{R T}-\lambda_{t m}^{R T}\right)+\sigma_{t n}^{+}-\sigma_{t n}^{-} \\
& +\sum_{n m \in \Lambda^{T S}} B_{n m}\left(\mu_{t, n m}^{+}-\mu_{t, m n}^{+}\right)+\left(\zeta_{t}\right)_{n=1} \\
& -\sum_{n m \in \Lambda^{T S}} B_{n m}\left(\mu_{t, n m}^{-}-\mu_{t, m n}^{-}\right)=0, \forall t, n \\
& \beta_{t}^{-} \geq 0 ; \quad \beta_{t}^{+} \geq 0, \quad \forall t \\
& \phi_{t g}^{-} \geq 0 ; \quad \phi_{t g}^{+} \geq 0, \quad \forall t, g \\
& \psi_{t g}^{-} \geq 0 ; \quad \psi_{t g}^{+} \geq 0, \quad \forall t, g \\
& \nu_{t d}^{-} \geq 0 ; \quad \nu_{t d}^{+} \geq 0, \quad \forall t, d \\
& \sigma_{t n}^{-} \geq 0 ; \quad \sigma_{t n}^{+} \geq 0, \quad \forall t, n \\
& \mu_{t, n m}^{-} \geq 0 ; \quad \mu_{t, n m}^{+} \geq 0, \quad \forall t, n m \in \Lambda^{T S} \\
& \sum_{t, g}\left(c_{g}^{U P} r_{t g}^{U P}-c_{g}^{D N} r_{t g}^{D N}\right)+\sum_{t} \lambda_{t I}^{D R T} P_{t I}^{D R T} \\
& +\sum_{t, d} \lambda_{t}^{S T} P_{t d}^{S T}+\sum_{t, g} \lambda_{t I}^{R T} P_{t g}^{G}+\sum_{t} \bar{P}^{D S}\left(\beta_{t}^{+}+\beta_{t}^{-}\right) \\
& +\sum_{t, g} \phi_{t g}^{+} R_{t g}^{U P}+\sum_{t, g} \psi_{t g}^{+} R_{t g}^{D N}+\sum_{t, n} \pi\left(\sigma_{t n}^{+}+\sigma_{t n}^{-}\right)
\end{aligned}
$$




$$
\begin{aligned}
& +\sum_{t, d} \nu_{t d}^{+} P_{t d}^{T S D}-\sum_{t, n} \lambda_{t n}^{R T}\left(\sum_{g_{(g: n) \in \mathcal{M}_{G}}} P_{t g}^{G}-P_{t d_{(d: n) \in \mathcal{M}_{D}}^{T S D}}\right) \\
& +\sum_{t, n m \in \Lambda^{T S}} \bar{P}_{n m}^{T S}\left(\mu_{t, n m}^{+}+\mu_{t, n m}^{-}\right)=0
\end{aligned}
$$

The recast single-level model is to minimize the PDISCO's objective, subject to PDISCO's constraints and the market's MPPDC constraints, as shown below:

$$
\begin{aligned}
& \underset{\Xi^{R T T} \cup \Xi^{P D I S C O} \cup \Xi^{\text {Dual }}}{\text { Minimize }}(2 \mathrm{a}) \\
& \text { s.t. } \\
& \text { PDISCO's problem constraints: }
\end{aligned}
$$

$(2 \mathrm{~b})-(2 \mathrm{~s})$

Real-time market problem MPPDC constraints:

$$
(1 \mathrm{~b})-(1 \mathrm{j}) \text { and }(3 \mathrm{a})-(3 \mathrm{l})
$$

The resulting non-linear model without complementarity can be solved by the commercial off-the-shelf large-scale non-linear optimization solver CONOPT3 [45].

\section{Case Studies}

To validate the effectiveness of the proposed bi-level model, a modified 14bus DL network [46] interconnected to a 9-bus TL network [47] is used to derive the PDISCO's strategic offers/bids. All cases are carried out on a 3.6-GHz Intel Core i7 processor based on a 64-bit Windows 7 system with 16 GB of RAM, and solved by CONOPT3 with GAMS 24.5.6 [45].

\subsection{Data}

For the PDISCO-operated 14-bus network: The capacities of the main substation $\bar{S}$ and each feeder $\bar{S}_{i j}$ are set to 400 MVA and 300 MVA, respectively. At the reference bus 1 , the voltage is fixed at 1 p.u.. The other buses' voltages range from 0.9 to 1.1 p.u.. The transformer tap ratio $\tau_{i}$ is imposed to 1 . 
Each compensator is with 0-50 MVar. The PDISCO manages DR portfolios and strategically trades in the real-time market with one time per hour (i.e. 24 times per day). The sales price $\lambda_{t}^{D S D}$ is shown in Table 1, which also includes the DL multiplier $\kappa_{t}$ to obtain the demand $P_{t l}^{D S D}, Q_{t l}^{D S D}$ on the base value described in [46]. The DL system-wide parameters $\Gamma$ and $\rho$ are set to 1.3 and 1 , respectively.

For the market-operated 9-bus network: The PDISCO's interconnection point is assumed to be at the TL bus 4 with a power injection limit of 300 MW. The line capacity is set to $500 \mathrm{MW}$. The TL multiplier $\gamma_{t}$ shown in Table I is used to obtain the day-ahead consumption $P_{t d}^{T S D}$ according to the base in [47. The real-time demand $P_{t d}^{T S R}$ is carried out on the TL network without the PDISCO's participation, and executed high real-time deviation $( \pm 10 \%)$ on $P_{t d}^{T S D}$. The real-time up/down regulation price $c_{g}^{U P} / c_{g}^{D N}$ is regarded as equal to the up/down reserve price. The outcomes of the day-ahead market, i.e., $P_{t g}^{G}$, $R_{t g}^{U P}, R_{t g}^{D N}$, and $\lambda_{t n}^{D A}$, can be generated by the clearing model (4). The related capacity limit $\bar{P}_{g}^{G}$, up/down reserve limit $\bar{R}_{g}^{U P} / \bar{R}_{g}^{D N}$, generation price $C_{g}^{G}$, and up/down reserve price $C_{g}^{U P} / C_{g}^{D N}$ of generator $g$ are shown in Table 2.

The DL and TL load-shedding prices $\left(\lambda_{t}^{S D}, \lambda_{t}^{S T}\right)$ are considered as 200 times the values of $\lambda_{t I}^{R T}$ and $\lambda_{t d}^{D A}$, respectively. Other parameters can be found in [46, 47. The price parameters mentioned above are estimated from the NordPool [48] prices.

\subsection{PDISCO trading strategies}

Trading in the real-time market, the PDISCO's strategic offering and bidding quantities are shown in Fig. 3, and the sequences of pricing strategies are presented in Fig. 4. The PDISCO's offers and bids constitute continuous curves. As expected, the PDISCO behaves as an active producer to provide virtual generation during hours 3, 11-13, 18 and 21, with lower offering prices (around $22 € / \mathrm{MW}$ ). In the remaining hours, purchasing power at higher prices (about $30-32 € / \mathrm{MW})$, the PDISCO performs as an active consumer. Unlike the dayahead unidirectional purchases, the hourly transactions between the PDISCO and the real-time market result in a bidirectional fashion. 
Table 1: Input Parameters for the PDISCO Trading in the Real-time Market

\begin{tabular}{c|c|c|c}
\hline $\begin{array}{c}t \\
{[\text { Hour }]}\end{array}$ & $\begin{array}{c}\lambda_{t}^{D S D} \\
{[€ / \mathrm{MW}]}\end{array}$ & $\kappa_{t}$ & $\gamma_{t}$ \\
\hline 1 & 8.0 & 2.4 & 0.3 \\
\hline 2 & 15.2 & 4.6 & 0.3 \\
\hline 3 & 16.0 & 4.8 & 0.3 \\
\hline 4 & 20.0 & 6.0 & 0.3 \\
\hline 5 & 28.0 & 8.4 & 0.8 \\
\hline 6 & 34.0 & 10.2 & 0.8 \\
\hline 7 & 40.0 & 12.0 & 0.9 \\
\hline 8 & 44.0 & 13.2 & 0.9 \\
\hline 9 & 46.0 & 13.8 & 1.0 \\
\hline 10 & 64.0 & 19.2 & 1.7 \\
\hline 11 & 72.0 & 21.6 & 1.8 \\
\hline 12 & 76.8 & 23.0 & 1.8 \\
\hline 13 & 72.0 & 21.6 & 1.7 \\
\hline 14 & 44.0 & 13.2 & 1.0 \\
\hline 15 & 36.0 & 10.8 & 1.0 \\
\hline 16 & 38.4 & 11.5 & 1.0 \\
\hline 17 & 44.0 & 13.2 & 1.0 \\
\hline 18 & 66.0 & 19.8 & 1.5 \\
\hline 19 & 68.0 & 20.4 & 1.6 \\
\hline 20 & 72.0 & 21.6 & 1.7 \\
\hline 21 & 76.0 & 22.8 & 1.8 \\
\hline 22 & 28.0 & 8.4 & 0.8 \\
\hline 23 & 16.8 & 5.0 & 0.5 \\
\hline 24 & 12.0 & 3.6 & 0.4 \\
\hline & & & \\
\hline 19 &
\end{tabular}

Table 2: Input Parameters for Generators

\begin{tabular}{c|c|c|c|c|c|c}
\hline No. & $\begin{array}{c}\bar{P}_{g}^{G} \\
{[\mathrm{MW}]}\end{array}$ & $\begin{array}{c}C_{g}^{G} \\
{[€ / \mathrm{MW}]}\end{array}$ & $\begin{array}{c}C_{g}^{U P} \\
{[€ / \mathrm{MW}]}\end{array}$ & $\begin{array}{c}C_{g}^{D N} \\
{[€ / \mathrm{MW}]}\end{array}$ & $\begin{array}{c}\bar{R}_{g}^{U P} \\
{[\mathrm{MW}]}\end{array}$ & $\begin{array}{c}\bar{R}_{g}^{D N} \\
{[\mathrm{MW}]}\end{array}$ \\
\hline 1 & 1500.0 & 27.0 & 30.0 & 22.0 & 500.0 & 500.0 \\
\hline 2 & 1200.0 & 29.0 & 32.0 & 24.0 & 300.0 & 300.0 \\
\hline 3 & 1200.0 & 36.0 & 38.0 & 30.0 & 300.0 & 300.0 \\
\hline
\end{tabular}




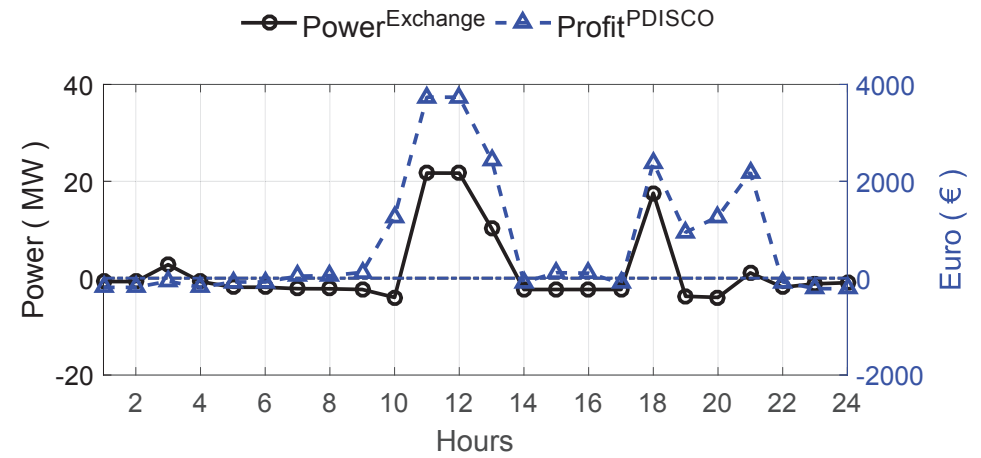

Figure 3: Results of the PDISCO's real-time offering/bidding quantities and profits.

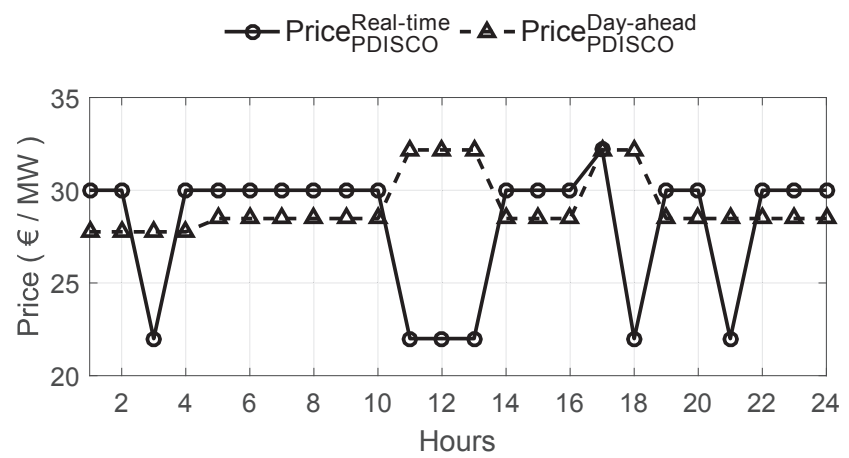

Figure 4: Results of the PDISCO's real-time offering/bidding prices. 
In addition, the PDISCO's trading profits are obtained and shown in Fig. 3. The real-time profits are recorded for comparison with power transactions. Observe that the profit peaks occur consistently with the offering power peaks. It is worth noting that, even during the bidding periods, the PDISCO's profits are still kept at around non-negative values with few volatilities. This prompts the PDISCO's daily profit to reach $€ 15808.35$. These results reveal that the PDISCO's trading strategies mentioned above are preferable and effective.

\subsection{Demand response}

As indicated in Section 2, the PDISCO's strategic behaviors originate from DR activations. Coordinating the trading strategies, the PDISCO simultaneously makes decisions on scheduling DR portfolios at individual demands. Each demand executes load shifting according to the schedules passed by the PDISCO.

The real-time consumption of the PDISCO is shown in Fig. 5. According to the real-time market clearing, the PDISCO consumes more in the low-price periods, resulting in a more rational load curve. Compared with the day-ahead predictions, large amounts of DR are motivated during the peak periods, i.e., hours 11-13 and 18, resulting in a $20 \%$ load reduction at each peak. In the realtime process, the reduced quantities are seen as the virtual generation of the PDISCO to render strategic offers. Thus, the high profits are achieved across these peak periods, corresponding to the strategies illustrated in Section 4.2 (Fig. 3 and 4 ).

At the distribution level, the numerical results of the real-time DR portfolio are also obtained ( 14 buses $\times 24$ hours). For illustration purposes, DL bus 3 is taken as an example to show the scheduled demand in Fig. 6, and to specify the hourly-based elasticity factor $\alpha_{t 3}$ in Table 3. We can observe that the load shifting occurs at hours $6,7,10-13,21$ and 22 , and the related $\alpha_{t 3}$ are in $[0$, 1). As addressed in Section 2.2, the shifted amounts spread out over other time periods, and are basically recognized as the higher consumptions characterized by $\alpha_{t 3}$ belonging to $[1,1.3]$. In particular, $\alpha_{t 3}$ reaches 1.3 (the elasticity limit) 


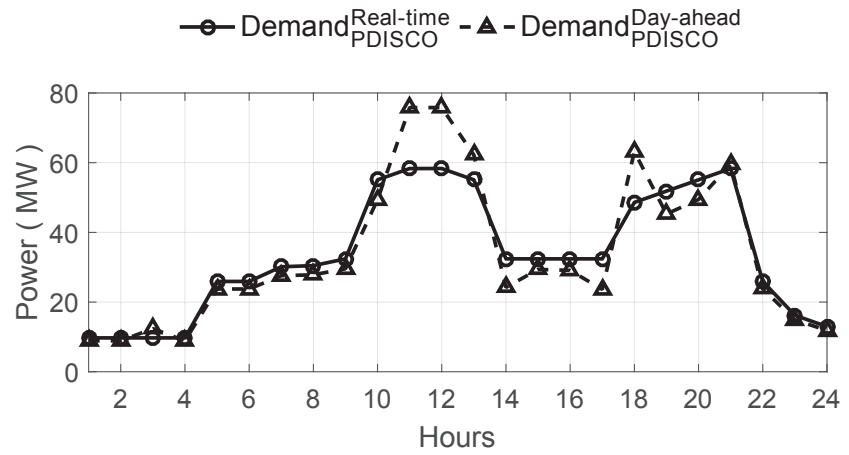

Figure 5: Results of the PDISCO's real-time consumptions.

at hours 1-5, 14, 16, 17 and 23. This implies that the PDISCO can launch preferential schemes to encourage the DL demands to consume extremely in the comparable low-price periods.

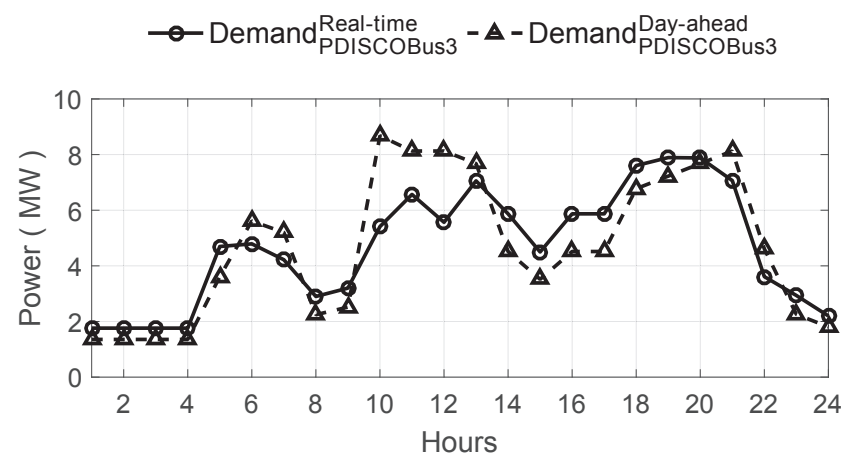

Figure 6: Results of the real-time DR at DL bus 3 .

Consequently, at each hour $t$, the real-time DR portfolios can be determined by the PDISCO and implemented by individual demands. To this end, the expected DR functions are explicitly achieved.

\subsection{Impact of elasticity limit $\Gamma$}

In order to quantify the DR's flexibility, the elasticity limit $\Gamma$ is used to enforce the DR upper bound at each demand $l$, as formulated in Section 3. Thus, $\Gamma$ is the key factor to simultaneously impact the real-time DL demand 
Table 3: Elasticity Factor of DL Bus 3

\begin{tabular}{c|c|c|c|c|c}
\hline $\begin{array}{c}t \\
{[\text { Hour }]}\end{array}$ & $\alpha_{t 3}$ & $\begin{array}{c}t \\
{[\text { Hour }]}\end{array}$ & $\alpha_{t 3}$ & $\begin{array}{c}t \\
{[\text { Hour }]}\end{array}$ & $\alpha_{t 3}$ \\
\hline 1 & 1.30 & 9 & 1.27 & 17 & 1.30 \\
\hline 2 & 1.30 & 10 & 0.62 & 18 & 1.12 \\
\hline 3 & 1.30 & 11 & 0.81 & 19 & 1.09 \\
\hline 4 & 1.30 & 12 & 0.68 & 20 & 1.03 \\
\hline 5 & 1.30 & 13 & 0.92 & 21 & 0.87 \\
\hline 6 & 0.85 & 14 & 1.30 & 22 & 0.78 \\
\hline 7 & 0.81 & 15 & 1.28 & 23 & 1.30 \\
\hline 8 & 1.29 & 16 & 1.30 & 24 & 1.22 \\
\hline
\end{tabular}

consumptions and the TL PDISCO strategies. Remaining the other parameters unchanged and setting consumption control factor $\rho=1$, given the deviation of $\Gamma$ by 0.1 per interval, the daily trading profit of the PDISCO and daily operation cost of the real-time market are obtained and shown in Fig. 7.

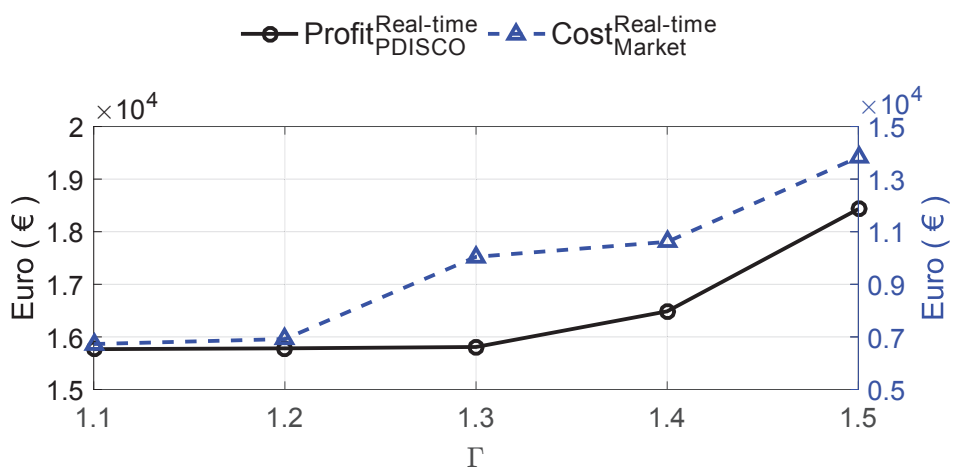

Figure 7: Impact of elasticity limit.

Observe that both the PDISCO's profit and the market's cost tend to be flat in the first two intervals, i.e. $\Gamma=1.1$ and 1.2. That means the PDISCO lacks DR elasticity and thus has very limited impact on the real-time trading within the TL market. In this situation, the major job of the PDISCO is to satisfy its daily consumption at a critical level. Prior to $\Gamma=1.3$, market cost rises sharply, while the PDISCO's daily profit increases progressively. This validates the PDISCO is willing to continuously trade within the real-time market under 
the premise that DRs provide more sufficient resources. With the $\Gamma$ increasing, extra availability of DRs causes a more competitive market. In this case, the PDISCO can behave more strategically in upwardly trading virtual generation and downwardly scheduling extensive DRs. Thus, the PDISCO's profit increases significantly when $\Gamma$ goes beyond 1.3. On the other hand, the market's cost also grows gradually. This implies that, on the basis of TL system operation constraints, the market operator has to find a trade-off between the power exchange with the PDISCO, the power purchases from individual generators, and the real-time balance of the TL demands.

Therefore, the higher $\Gamma$ is preferable for the PDISCO, but unexpected for the market.

\subsection{Impact of consumption control factor $\rho$}

As illustrated in Section 3, the PDISCO's strategic offers/bids highly depend on its demand capacity. We further focus on the consumption impacts for the PDISCO's strategies by resetting the demand availability in additional cases. The elasticity limit is set at $\Gamma=1.2$. Thus, the previous discussions are seen as Case $1(\rho=1)$. With the other parameters unchanged, the daily consumption control factor $\rho$ is adjusted by 0.1 per case to capture its characteristics in real-time trading, as shown in Fig. 8.

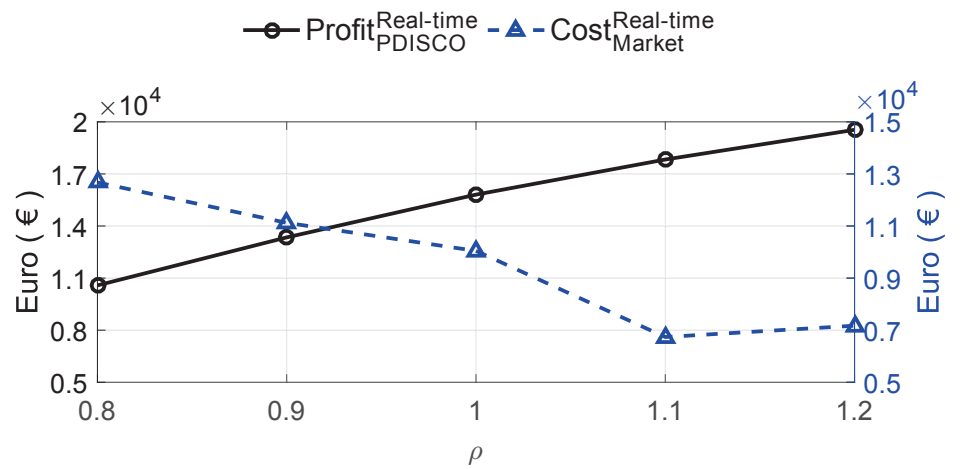

Figure 8: Impact of consumption control factor.

1) Case 2: $\rho<1$. In this situation, we set $\rho$ at 0.8 and 0.9 , respectively. 
Compared with Case 1, the PDISCO's daily profit inevitably declines, since the consumption drops significantly while the DL sale price $\lambda_{t}^{D S D}$ is fixed. The occasional power exchange slightly compensates the PDISCO's revenue, but remarkably increases the market's cost for purchasing regulation power. The lower $\rho$ definitely worsens the performance of both the PDISCO and the realtime market.

2) Case 3: $\rho>1$. On the basis of Case $1, \rho$ values of 1.1 and 1.2 are considered. The higher DL demand capacity yields more offers and fewer bids from the PDISCO in real-time trading, thus, its profit keeps increasing consistently. When $\rho$ exceeds 1.1, the market's cost stops decreasing and turns upward. The reason is that when $\rho<1.1$, the market benefits more from the low-price offers rendered by the PDISCO versus comparatively high regulation prices; however, when $\rho>1.1$, the excessive offers of the PDSICO become burdens for the market operation.

In terms of the discussions above, the PDISCO desires a higher $\rho$, while the market needs a superior $\rho$ (e.g., $\rho=1.1$ ).

\section{Conclusion}

This paper presents a bi-level model for a PDISCO strategically trading within the TL real-time market by scheduling the DL DR portfolios. The elasticity of each DL demand is used as the DR resource to achieve load shifting in the presented real-time trading framework. To maximize the PDISCO's profit, the proposed model can continuously generate strategic offers and bids. Interrelated with the PDISCO's strategies, the operation cost of the real-time market is minimized. The proposed bi-level model is finally recast as an MPEC with

the primal-dual approach. The numerical results and impact analyses verify the effectiveness of the proposed methodology.

To implement the correlated TL-DL trading structure, the exiting TL realtime market may consider a PDISCO as a bidirectional transactor, while the PDISCO regards the TL market and the DL DRs as power providers. In par- 
ticular, when behaving as an active producer, the PDISCO could be seen as a large-scale stochastic power producer (e.g., wind farm) to participate in the real-time market.

\section{Appendix}

\section{Day-ahead market clearing model}

The day-ahead market is cleared by (4). The objective function (4a) is to minimize the minus (maximize) TL social welfare, i.e., the generation and reserve cost of individual generators minus the bids of TL demands.

$$
\begin{aligned}
& \underset{\Xi D A T}{\operatorname{Minimize}} \sum_{t, g}\left(C_{g}^{G} P_{t g}^{G}+C_{g}^{U P} R_{t g}^{U P}+C_{g}^{D N} R_{t g}^{D N}\right) \\
& -\sum_{t, d} \lambda_{t d}^{T S D} P_{t d}^{T S D}
\end{aligned}
$$

s.t.

$$
\begin{aligned}
& \sum_{(g: n) \in \mathcal{M}_{G}} P_{t g}^{G}-P_{t d}^{T S D}=\sum_{n m \in \Lambda^{T S}} B_{n m}\left(\theta_{t n}^{0}-\theta_{t m}^{0}\right) \\
& : \lambda_{t n}^{D A}, \forall t, n_{(d: n) \in \mathcal{M}_{D}} \\
& 0 \leq P_{t g}^{G}+R_{t g}^{U P} \leq \bar{P}_{t g}^{G}, \forall t, g \\
& 0 \leq P_{t g}^{G}-R_{t g}^{D N}, \forall t, g \\
& 0 \leq R_{t g}^{U P} \leq \bar{R}_{g}^{U P}, \forall t, g \\
& 0 \leq R_{t g}^{D N} \leq \bar{R}_{g}^{D N}, \forall t, g \\
& -\bar{P}_{n m}^{T S} \leq B_{n m}\left(\theta_{t n}^{0}-\theta_{t m}^{0}\right) \leq \bar{P}_{n m}^{T S}, \forall t, n m \in \Lambda^{T S} \\
& -\pi \leq \theta_{t n}^{0} \leq \pi, \forall t, n \\
& \theta_{t 1}^{0}=0, \forall t, n=1
\end{aligned}
$$

where $\Xi^{D A T}=\left\{P_{t g}^{G}, R_{t g}^{U P}, R_{t g}^{D N}, \theta_{t n}^{0}\right\}$ is the variable set of the day-ahead market clearing problem.

On the basis of DC power flow, at each time $t$ : Constraints (4b) represent the power balance at each TL bus. Constraints (4c), (4d), (4e) and (4f) impose the capacity limits of the up/down reserve for generator $g$. Constraints (4g) 
indicate the capacity limits of the TL line $n-m$. Constraints (4h) express the

range of the day-ahead TL voltage angles $\theta_{t n}^{0}$, and constraints (4i) set TL bus 1 as the reference bus.

\section{Acknowledgments}

The authors would like to acknowledge the support of the Research Council of Norway with project 255209, the Danish iPower project 10-095378, and the U.S. Department of Energy (DOE)'s Office of Electricity Delivery and Energy Reliability.

\section{References}

[1] Q. Wang, C. Zhang, Y. Ding, G. Xydis, J. Wang, J. Østergaard, Review of real-time electricity markets for integrating distributed energy resources and demand response, Appl. Energy 138 (2015) 695-706. doi:10.1016/j. apenergy.2014.10.048.

[2] S. Nolan, M. O'Malley, Challenges and barriers to demand response deployment and evaluation, Appl. Energy 152 (2015) 1-10. doi:10.1016/j. apenergy.2015.04.083.

[3] C. Zhang, Q. Wang, J. Wang, P. Pinson, J. M. Morales, J. Østergaard, Real-time procurement strategies of a proactive distribution company with aggregator-based demand response, IEEE Trans. Smart Grid (2016, in press) $1-10$.

[4] C. Zhang, Market design and strategy making for proactive distribution grid with DERs, Technical University of Denmark, Denmark, 2015.

[5] C. Chen, J. Wang, S. Kishore, A distributed direct load control approach for large scale residential demand response, IEEE Trans. on Power Syst. 29 (5) (2014) 2219-2228. doi:10.1109/TPWRS.2014.2307474. 
[6] NewYorkState, Reforming the energy vision, NYS Department of Public Service.

[7] Z. Zhou, F. Zhao, J. Wang, Agent-based electricity market simulation with demand response from commercial buildings, IEEE Trans. Smart Grid 2 (4) (2011) 580-588. doi:10.1109/TSG.2011.2168244

[8] C. Li, X. Yu, W. Yu, G. Chen, J. Wang, Efficient computation for sparse load shifting in demand side management, IEEE Trans. Smart Grid (2016) 1-12doi:10.1109/TSG.2016.2521377.

[9] Z. Wang, B. Chen, J. Wang, J. Kim, M. M. Begovic, Robust optimization based optimal DG placement in microgrids, IEEE Trans. Smart Grid 5 (5) (2014) 2173-2182. doi:10.1109/TSG.2014.2321748

[10] Z. Wang, B. Chen, J. Wang, C. Chen, Networked microgrids for self-healing power systems, IEEE Trans. Smart Grid 7 (1) (2015) 310-319. doi:10. 1109/TSG.2015.2427513.

[11] C. Zhang, Q. Wang, J. Wang, M. Korpås, P. Pinson, J. Østergaard, M. E. Khodayar, Trading strategies for distribution company with stochastic distributed energy resources, Appl. Energy 177 (2016) 625-635. doi: $10.1016 / \mathrm{j}$. apenergy.2016.05.143

[12] D. Neves, A. Pina, C. A. Silva, Demand response modeling: A comparison between tools, Appl. Energy 146 (2015) 288-297. doi:10.1016/j. apenergy.2015.02.057.

[13] C. Zhang, Q. Wang, J. Wang, P. Pinson, J. Østergaard, Real-time trading strategies of proactive DISCO with heterogeneous DG owners, IEEE Trans. Smart Grid (2016, in press) 1-10.

[14] A. Safdarian, M. Fotuhi-Firuzabad, M. Lehtonen, Integration of price-based demand response in DisCos' short-term decision model, IEEE Trans. Smart Grid 5 (5) (2014) 2235-2245. doi:10.1109/TSG.2014.2317502. 
[15] D. T. Nguyen, M. Negnevitsky, M. De Groot, Market-based demand response scheduling in a deregulated environment, IEEE Trans. Smart Grid 4 (4) (2013) 1948-1956. doi:10.1109/TSG.2013.2258410.

[16] C. Bartusch, K. Alvehag, Further exploring the potential of residential demand response programs in electricity distribution, Appl. Energy 125 (2014) 39-59. doi:10.1016/j. apenergy.2014.03.054.

[17] F. Kamyab, M. Amini, S. Sheykhha, M. Hasanpour, M. M. Jalali, Demand response program in smart grid using supply function bidding mechanism, IEEE Trans. Smart Grid 7 (3) (2016) 1277-1284. doi:10.1109/TSG . 2015. 2430364

[18] S. A. Gabriel, A. J. Conejo, J. D. Fuller, B. F. Hobbs, C. Ruiz, Complementarity modeling in energy markets, Springer, New York, 2012.

[19] H. Haghighat, S. W. Kennedy, A bilevel approach to operational decision making of a distribution company in competitive environments, IEEE Trans. on Power Syst. 27 (4) (2012) 1797-1807.

[20] S. D. Manshadi, M. E. Khodayar, A hierarchical electricity market structure for the smart grid paradigm, IEEE Trans. Smart Grid (2015) 1-10.

[21] D. T. Nguyen, H. T. Nguyen, L. B. Le, Dynamic Pricing Design for Demand Response Integration in Power Distribution Networks, IEEE Trans. Power Syst. (2016) 1-16doi:10.1109/TPWRS.2015.2510612.

[22] R. Palma-Behnke, J. L. Cerda A., L. S. Vargas, A. Jofré, A distribution company energy acquisition market model with integration of distributed generation and load curtailment options, IEEE Trans. Power Syst. 20 (4) (2005) 1718-1727.

[23] H. Li, Y. Li, Z. Li, A multiperiod energy acquisition model for a distribution company with distributed generation and interruptible load, IEEE Trans. Power Syst. 22 (2) (2007) 588-596. doi:10.1109/TPWRS.2007.894862. 
[24] J. Wang, M. Shahidehpour, Z. Li, A. Botterud, Strategic generation capacity expansion planning with incomplete information, IEEE Trans. Power Syst. 24 (2) (2009) 1002-1010. doi:10.1109/TPWRS.2009.2017435.

[25] C. Ruiz, A. J. Conejo, Pool strategy of a producer with endogenous formation of locational marginal prices, IEEE Trans. on Power Syst. 24 (4) (2009) 1855-1866. doi:10.1109/TPWRS.2009.2030378.

[26] L. Baringo, A. J. Conejo, Offering strategy of wind-power producer: a multi-stage risk-constrained approach, IEEE Trans. on Power Syst. 31 (2) (2015) 1420-1429. doi:10.1109/TPWRS.2015.2411332.

[27] R. Fernández-Blanco, J. M. Arroyo, N. Alguacil, Network-constrained dayahead auction for consumer payment minimization, IEEE Trans. Power Syst. 99 (2) (2013) 1-11. doi:10.1109/TPWRS.2013.2284283

[28] S. J. Kazempour, A. J. Conejo, C. Ruiz, Strategic bidding for a large consumer, IEEE Trans. Power Syst. 30 (2) (2015) 848-856. doi:10.1109/ TPWRS.2014.2332540

[29] Z. Wang, B. Chen, J. Wang, M. M. Begovic, C. Chen, Coordinated energy management of networked microgrids in distribution systems, IEEE Trans. Smart Grid 6 (1) (2015) 45-53.

[30] G. E. Asimakopoulou, A. L. Dimeas, N. D. Hatziargyriou, Leader-follower strategies for energy management of multi-microgrids, IEEE Trans. Smart Grid 4 (4) (2013) 1909-1916. doi:10.1109/TSG.2013.2256941.

[31] D. Papadaskalopoulos, G. Strbac, Decentralized participation of flexible demand in electricity markets - part I: market mechanism, IEEE Trans. Power Syst. 28 (4) (2013) 3658-3666. doi:10.1109/TPWRS.2013.2245686

[32] D. Papadaskalopoulos, G. Strbac, P. Mancarella, M. Aunedi, V. Stanojevic, Decentralized participation of flexible demand in electricity markets - part II: application with electric vehicles and heat pump systems, IEEE Trans. Power Syst. 28 (4) (2013) 3667-3674. doi:10.1109/TPWRS.2013.2245687. 
[33] M. Rahimiyan, L. Baringo, A. J. Conejo, Energy management of a cluster of interconnected price-responsive demands, IEEE Trans. Power Syst. 29 (2) (2014) 645-655. doi:10.1109/TPWRS.2013.2288316.

[34] R. Fernandez-Blanco, J. M. Arroyo, N. Alguacil, X. Guan, Incorporating price-responsive demand in energy scheduling based on consumer payment minimization, IEEE Trans. on Smart Grid 7 (2) (2015) 817-826. doi: 10.1109/TSG.2015.2427584

[35] M. Parvania, M. Fotuhi-Firuzabad, M. Shahidehpour, ISO's optimal strategies for scheduling the hourly demand response in day-ahead markets, IEEE Trans. Power Syst. 29 (6) (2014) 2636-2645. doi:10.1109/TPWRS. 2014. 2316832

[36] Y. Xu, N. Li, S. H. Low, Demand response with capacity constrained supply function bidding, IEEE Trans. on Power Syst. 31 (2) (2016) 1377-1394.

[37] D. Wang, S. Parkinson, W. Miao, H. Jia, C. Crawford, N. Djilali, Hierarchical market integration of responsive loads as spinning reserve, Appl. Energy 104 (2013) 229-238. doi:10.1016/j . apenergy.2012.10.054.

[38] M. Mazidi, H. Monsef, P. Siano, Incorporating price-responsive customers in day-ahead scheduling of smart distribution networks, Appl. Energy 178 (2016) 929-942. doi:10.1016/j.apenergy.2016.06.016.

[39] T. Broeer, J. Fuller, F. Tuffner, D. Chassin, N. Djilali, Modeling framework and validation of a smart grid and demand response system for wind power integration, Appl. Energy 113 (2014) 199-207. doi:10.1016/j .apenergy. 2013.06 .058

[40] N. Mahmoudi, T. K. Saha, M. Eghbal, Modelling demand response aggregator behavior in wind power offering strategies, Appl. Energy 133 (2014) 347-355. doi:10.1016/j.apenergy . 2014.07.108. 
[41] J. L. Mathieu, S. Koch, D. S. Callaway, State estimation and control of electric loads to manage real-time energy imbalance, IEEE Trans. on Power Syst. 28 (1) (2013) 430-440. doi:10.1109/TPWRS.2012.2204074.

[42] Y. Kitapbayev, J. Moriarty, P. Mancarella, Stochastic control and real options valuation of thermal storage-enabled demand response from flexible district energy systems, Appl. Energy 137 (2015) 823-831. doi:10.1016/ j.apenergy.2014.07.019.

[43] J. Kondoh, N. Lu, D. J. Hammerstrom, An evaluation of the water heater load potential for providing regulation service, IEEE Trans. on Power Syst. 26 (3) (2011) 1309-1316.

[44] J. M. Arroyo, Bilevel programming applied to power system vulnerability analysis under multiple contingencies, IET Gener. Transm. Distrib. 4 (2) (2010) 178-190. doi:10.1049/iet-gtd.2009.0098.

[45] GAMS, [Online]. Available: http://www.gams.com/.

[46] F. R. Alonso, D. Q. Oliveira, A. C. Zambroni de Souza, Artificial immune systems optimization approach for multiobjective distribution system reconfiguration, IEEE Trans. on Power Syst. 30 (2) (2015) 840-847. doi:10.1109/TPWRS.2014.2330628

[47] A. Jamehbozorg, G. Radman, Small signal analysis of power systems with wind and energy storage units, IEEE Trans. on Power Syst. 30 (1) (2015) 298-305. doi:10.1109/TPWRS.2014.2321711.

[48] Nordpool, [Online]. Available: http://www.nordpoolspot.com/. 